Article

\title{
Estimation of the Lifespan Distribution of Gold Nanoparticles Stabilized with Lipoic Acid by Accelerated Degradation Tests and Wiener Process
}

\author{
Betania Sánchez-Santamaría ${ }^{1}$, Boris Mederos ${ }^{1}$, Delfino Cornejo-Monroy ${ }^{1}$, Rey David Molina-Arredondo ${ }^{1}$ and Víc- $^{-}$ \\ tor Castaño ${ }^{*}$ \\ 1 Instituto de Ingeniería y Tecnología (IIT), Universidad Autónoma de Ciudad Juárez (UACJ), 32310 \\ Chihuahua, México; betania_sanchez@hotmail.com (B.S.-S.); boris.mederos@uacj.mx (B.M.); delfino.cor- \\ nejo@uacj.mx (D. C.-M.); rey.molina@uacj.mx (R.D.M.-A.); \\ 2 Centro de Física Aplicada y Tecnología Avanzada, Universidad Nacional Autónoma de México (UNAM); \\ vmcastano@unam.mx (V.C.-M.) \\ * Correspondence: vmcastano@unam.mx
}

\begin{abstract}
Accelerated degradation tests (ADT) are widely used in the manufacturing industry to obtain information on the reliability of components and materials, through degrading the lifespan of the product by applying an acceleration factor which causes damage to the material. The main objective is to obtain fast information which is modeled to estimate the characteristics of the material life under normal conditions of use and to save time and expenses. The purpose of this work is to estimate the lifespan distribution of gold nanoparticles stabilized with lipoic acid (GNPs@LA) through accelerated degradation tests applying sodium chloride $(\mathrm{NaCl})$ as an acceleration factor. For this, the synthesis of GNPs@LA was carried out, a constant stress ADT (CSADT) was applied, and the non-linear Wiener process was proposed with random effects, error measures and different covariability for the adjustment of the degradation signals. The information obtained with the test and analysis allows us to obtain the life distribution in GNPs@LA, the results make possible to determine the guaranteed time for a possible commercialization and successful application based on the stability of the material. In addition, for the evaluation and selection of the model, the Akaike and Bootstraping criteria were used.
\end{abstract}

Keywords: accelerated degradation tests; gold nanoparticles; life distribution; Wiener process

\section{Introduction}

Accelerated degradation tests (ADT) are an effective tool for evaluating the reliability of materials through the analysis of degradation data, these tests consist of degrading the life of the product by applying a factor that accelerates degradation, thereby obtaining degradation data, which are used to estimate the life distribution of the material under normal conditions of use and at the same time minimize costs and times involved in the test, obtaining good material life data.

For recently created materials, current studies have adopted an ADT in the evaluation of reliability based on the Wiener process. The Wiener process is frequently found in practice as it provides a satisfactory and flexible description on degradation data obtained after having performed an ADT [1,2].

Nowadays, nanoscience and nanotechnology develop highly innovative materials and products with the ability to revolutionize life as we know it. These nanomaterials, like any other material, show deterioration that involves a very complex interaction between stress, time, and the environment, eventually causing the failure of the product [3]. In this 
way, at any technological field, knowing the useful life of the products is required for the successful application.

For nanostructured materials, to our knowledge, it has not been determined the life span within an appropriate test time, and the studies found in the literature do not use test methods and degradation analysis in order to have reliable information on the life's nanomaterials over the time, moreover, some studies report the need for regulatory reforms to improve supervision of nanomaterials throughout its life cycle [4,5]. Faced with this condition, there is a wide opportunity of using the ADTs for nanostructured materials to estimate the useful life, as well as, to contribute for the regulation of these materials.

A material of great interest at the nanoscale is gold, which is probably one of the most fascinating material due to its physical and chemical properties at the nano-scale [6,7]; gold nanostructures (GNPs) have shown potential applications in many research areas. In medicine, ultra-small nanoparticles below $5 \mathrm{~nm}$ have unique advantages in the human body due to their relatively rapid clearance, good absorption, and favorable interaction with radiation [6,8]. For example, gold nanostructures have been tested as sensors [9] capable of detecting certain diseases such as cancer [10], SARS-CoV-2 [11], Alzheimer's and Salmonella $[9,10]$, also they were utilized as chemical carriers $[12,13]$ and as theragnostic agents $[10,14]$.

For the successful application, there is evidence that the stability of gold nanoparticles must be well known in order to reach the desired tissues or cells [15], overcoming the limitations of biological barriers to diagnose ${ }^{3}$ and treat deep targets $[8,16]$. Some variables that influence the alteration of stability are the $\mathrm{pH}$ [17] of the medium as well as the presence of $\mathrm{NaCl}[18]$.

GNPs@LA analyzed in this study are spherical of $2.5 \mathrm{~nm}$, highly stable in colloid as well as powder; they are stabilized with lipoic acid which prevents agglomeration and creates functional groups for bio-conjugation, and also do not present toxic effects at the cellular level based on ISO 10993-5 [19]. However, there are no studies that allow us to determine the lifetime of the nanomaterial. The purpose of this study is to estimate the failure rate and useful life of GNPs@LA through ADT relied on Wiener process applying $\mathrm{NaCl}$ as an acceleration factor. The proposed methodology is an important contribution to provide nanomaterials guarantees and opens the door for the development of further research.

Summarizing, the main contributions are:

- A methodology based on accelerated degradation testing and Wiener process to estimate the useful life of GNPs@LA.

- $\quad$ Estimation of the failure rate GNPs@LA using $\mathrm{NaCl}$ as a degradation factor.

- A methodology which relies on non-linear Wiener process with random effects, error measures and different covariability to determine the useful life of the GNPs@LA.

- A rigorous statistical analysis to determine the most appropriated Wiener degradation model.

The rest of this paper is structured as follows: section 2 provides a general description of synthesis and stability of GNP@LA, an explanation of the accelerated degradation model with the Wiener process. Also, it is presented a statistical inference framework based on maximum likelihood estimation (MLE) method to estimate the parameters of the life distribution. At the end of this section, the statistical framework is applied to our specific degradation problem. Section 3 explores and compares the specified degradation models on our degradation data showing which is the more appropriate model to estimate life distribution. Finally, section 4 gives the conclusions of our work.

\section{Materials and Methods}

\subsection{Synthesis of GNPs@LA}


Based on the bottom-up approach, particularly the colloidal method; gold nanoparticles with an average size of $2.5 \mathrm{~nm}$ were synthetized and stabilized using gold chloride as a precursor, sodium borohydride as a reducer and lipoic acid as a stabilizer, following the methodology reported by Cornejo-Monroy et al [19].

\subsection{Stability of GNPs@LA}

Gold colloid stability implies that solid nanoparticles do not settle or aggregate at a significant speed [20]. When nanoparticles lose their stability by aggregation, particle size increase and creates agglomerates, losing their interesting properties.

One way to measure how these properties are affected according to its size is through their characterization by UV-Vis spectroscopy [21], which is a simple and reliable method to monitor the stability of the gold colloids. As the nanoparticles become destabilized, the original characteristic peak will decrease in intensity due to the depletion of stable nanoparticles, and often the peak will be broaden to longer wavelengths due to the formation of aggregates or agglomerates [22]. The shape and peak position of UV-Vis spectra are related to the morphology and size of the nanoparticle, as well as, the dispersion/aggregation of gold colloids [23]. Gold colloids present electronic transitions of bands in the visible range between $450 \mathrm{~nm}$ and $550 \mathrm{~nm}$. Therefore, some visible wavelengths are absorbed, emitting a characteristic color which can be characterized and related to morphological changes in the nanomaterial [23]. In figure 1, J. Martínez et al [24] shows different sizes of gold nanoparticles as a function of the characteristic peak of the plasmon band, it can be observed that their characteristic peak is red-shift as the GNPs diameter increases. This is because the optical properties of gold nanoparticles change when the particles aggregate and the conduction electrons near the surface of each particle are delocalized and shared among neighboring nanoparticles [25]. When this occurs, the surface plasmon resonance shifts to lower energies, causing the characteristic absorption and scattering peak shift to longer wavelengths [24].

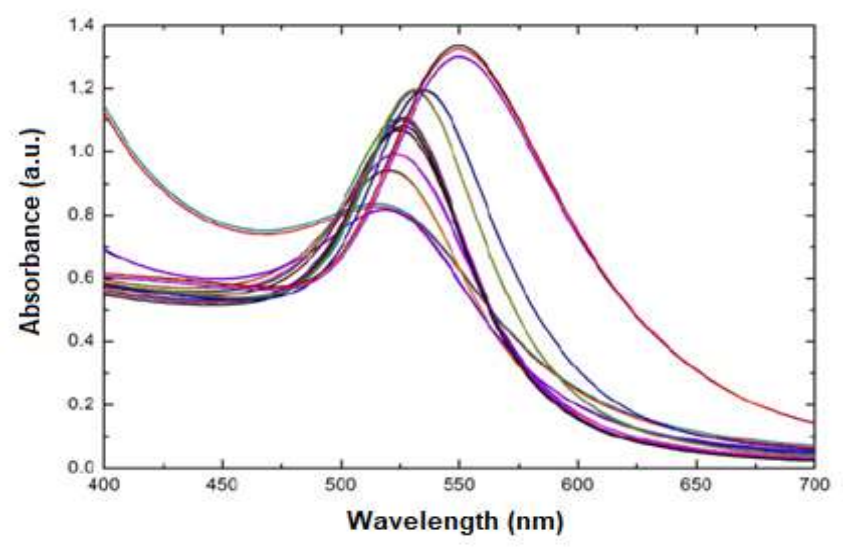

Figure 1. UV-Vis absorption spectra of GNPs of 5, 10, 15, 20, 30, 40, 50, 60 and $80 \mathrm{~nm}$ in diameter from bottom to top, respectively. Taken from J. Martínez et al [24].

For this study, and as an example to apply an ADT analysis, to determine the failure rate and useful life of GNPs@LA we considered that gold nanoparticles with UV-Vis spectra peak greater than $525 \mathrm{~nm}$ failed.

\subsection{Accelerated Degration Model}

One objective of the reliability analysis is to estimate the useful life of the product through the life distribution. To obtain the life distribution of a product using degradation data, the central step is to set up a model that describes the degradation process, called an 
accelerated degradation model. An accelerated degradation model is the combination of an accelerated model and a degradation model based on physics and statistical models.

An accelerated model shows the relationship between life and effort to establish the connection between degradation data and product life, it is essential to establish a suitable probability model to describe the behavior of collected degradation data, also known as degradation trajectories. There are two types of degradation models that are commonly used, which are the general trajectory models and the stochastic models.

General trajectory models are described as simple and easy to use, but they lack the ability to capture system dynamics. In contrast, stochastic models have great potential to capture random dynamics within degradation processes. The Wiener process, the Gamma process, and the Inverse Gaussian process are three common stochastic processes that have received many applications in degradation modeling $[9,26]$. However, it should be noted that both the Gamma process and the Inverse Gaussian process are only suitable for modeling monotonous degradation trajectories. In comparison, the Wiener process is applicable to non-monotonous degradation processes that are frequently encountered in practice as it provides a satisfactory and flexible description in the degradation data [2]. The Winner process have been widely applied to degradation data analysis for example to light emitting diodes [27], fatigue of metals [28], aluminum reduction cells [29], microelectromechanical systems [30], among others.

\subsection{Wiener Degradation Process}

Since components of a systems deteriorate over time and fail when the degradation level reach certain threshold. The Degradation information can be measured in a nondestructive way, after which an appropriated degradation model is chosen to describe the process through analysis of the data. Among the degradation models, the Wiener process with positive drift is a well stablished method due to its mathematical properties, it is expressed as follows,

$$
X(t)=\lambda \Lambda(t)+\sigma B(\Lambda(t))
$$

where $\Lambda(t)$ represents the transformed time scale and it is a monotonic continuous function that explains the non-linearization of the data, typical examples are: $\Lambda(t)=\mathrm{e}^{b t}, \Lambda(t)=$ blnt, $\Lambda(t)=t^{b}$, where $0<b<1$ is a parameter to be estimated [31]. The parameters $\lambda$ and $\sigma$ stand for the drift and diffusion parameter, respectively. $B(t)$ corresponds to the standard Brownian motion which satisfies the following properties:

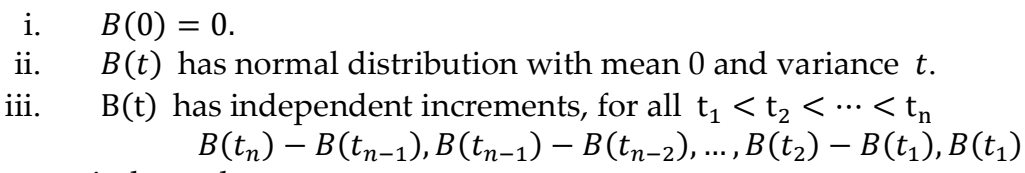
are independent.

iv. $\{B(t), t \geq 0\}$ has stationary increments. That is, the distribution of $B(t+s)-$ $B(t)$ does not depent of $t$.

from the above properties it can be deduced that random vector $\left(B\left(t_{1}\right), B\left(t_{2}\right), \ldots, B\left(t_{n}\right)\right) \sim \mathcal{N}(0, \Sigma)$, where the covariance matrix $\Sigma=\left(\min \left\{t_{i}, t_{j}\right\}\right)_{i, j}, i, j=$ $1, \ldots, n$. The previous properties on $B(t)$ entails that the Wiener degradation process $X(t)$ has the same properties of the Brownian motion $B(t)$ but the first property. Additional is straightforward to see that $X(t) \sim \mathcal{N}\left(\lambda \Lambda(t), \sigma^{2} \Lambda(t)\right)$.

In the process of determine the degradation values, measurement errors are inescapably introduced by cause of measurement instrument errors, operation errors, among other factors. Thus, the obtained degradation values are not the real ones, consequently 
an error of measurements $\epsilon$, with $\epsilon \sim \mathcal{N}\left(0, \sigma_{\epsilon}^{2}\right)$ is introduced, leading to an observe degradation process as follows

$$
Y(t)=X(t)+\epsilon
$$

Since stress factors such as voltage, humidity, temperature, vibration, etc. affect the performance of the degradation process, then an acceleration model can be used to integrate the covariate into the Wiener process. The most common way to incorporate the acceleration model into the Wiener process is to consider some model parameters as a covariate function which is typically called a link function $h(\cdot)$. The choice of the form of this function will depend on the way the acceleration factor influences the model parameters. Some accelerated models are Arrhenius model, Inverse power model, Eyring model, linear and quadratic model, which are summarized in the following Table 1.

Table 1. Acceleration models and its link function

\begin{tabular}{cc}
\hline Acceleration model & Link function \\
\hline Arrhenius Model & $h\left(S_{k}\right)=\exp \left(-\frac{\beta}{S_{k}}\right)$ \\
Inverse Power & $h\left(S_{k}\right)=\exp \left(-\beta \ln \left(S_{k}\right)\right)$ \\
Linear Model & $h\left(S_{k}\right)=\exp \left(-\beta\left(S_{k}\right)\right)$ \\
Quadratic Model & $h\left(S_{k}\right)=\exp \left(-\beta_{1}\left(S_{k}\right)+\beta_{2}\left(S_{k}\right)^{2}\right)$ \\
\hline
\end{tabular}

Therefore, the acceleration model of the drift parameter and diffusion parameter depends on the stress factor by means of the link function as follows

$$
\lambda_{k}=\eta h\left(S_{k}\right), \quad \sigma_{k}^{2}=\kappa h\left(S_{k}\right),
$$

where $S_{k}$ denotes the stress level and $\eta$ represents a variability parameter and $k$ is a constant factor associated to the diffusion. From now on $h\left(S_{k}\right)$ will be represented as $h_{k}$.

It is common to find differences between the degradation trajectories from unit to unit of the population. This type of differences is the result of non-observable random effects. In order to express this, some model parameter will be specifics for each unity, obtaining a process with certain parametric distribution [28]. Because it is difficult to consider both $\lambda_{k}$ and $\sigma_{k}$ randomly, the model drift parameter $\lambda_{k}$ will be specific for each unit and follows a normal distribution, on the other hand the parameter $\sigma_{k}$ will be taken as a constant, Peng and Tseng [32]. Si et al [33,34] and Tsai et al [35]. Thus, it is assumed that the variability parameter $\eta$ is a random variable with normal distribution $\mathcal{N}\left(\mu_{\eta}, \sigma_{\eta}^{2}\right)$. Putting all this together into (2), it is obtained

$$
Y_{k}(t)=Y\left(t \mid S_{k}\right)=\eta h_{k} \Lambda(t)+\sqrt{\kappa h_{k}} B(\Lambda(t))+\epsilon,
$$

where (4) models the ADT with random effects, error measurements and covariates. There are studies that configure more than one variant in the Wiener process, for instance, Li Sun et al [36] describe a methodology for the model and estimation of parameters through a constant stress ADT (CSADT) applying the non-linear Wiener process, with covariates, random effects, and measurement errors. A CSADT is a test plan consisting of three to four levels of tests with different proportions of units in each one, where mainly at the low effort level more samples run than at a high level and this type of plan can provide accurate estimates for an ADT. Following the notation in [36], the increasing applied stress level are $\mathrm{S}_{1}, \ldots \mathrm{S}_{\mathrm{k}}, \ldots \mathrm{S}_{\mathrm{K}}$, where $K$ denotes the maximum stress level. Also, there are $N_{k}$ units 
tested under each constant stress $S_{k}$ and each unit is measured $M_{k i}$ times at the $k$ stress level with $i=1,2, \ldots, N_{k}$. The transformed time will be expressed as $\omega_{k i j}=\Lambda\left(t_{k i j}\right)$ with $j=1,2, \ldots, M_{k i}$.

Therefore, the degradation observed under the Wiener process with its four variants is shown as follows

$$
y_{k i j}\left(\omega_{k i j} \mid S_{k}\right)=\eta_{i} h_{k} \omega_{k i j}+\sqrt{\kappa h_{k}} B\left(\omega_{k i j}\right)+\varepsilon_{k i j},
$$

with $\eta_{i} \sim N\left(\mu_{\eta}, \sigma_{\eta}^{2}\right), \varepsilon_{k i j} \sim N\left(0, \sigma_{\varepsilon}^{2}\right)$ and $h_{k}$ the link function of the accelerated model. The model (5) comprises the following set of unknown parameters,

$$
\boldsymbol{\Theta}=\left\{\mu_{\eta}, \sigma_{\eta}^{2}, \kappa, \beta, \sigma_{\varepsilon}^{2}\right\}
$$

that will be estimated in the next section.

2.5. Statistical Inference of the Wiener Degradation Process, parameters estimation and life distribution on ADT Data

In the previous section a model for CSADT was formulated in (5), in order to estimate the unknown parameters set $\widetilde{\boldsymbol{\Theta}}$ let consider the following vectors $\boldsymbol{T}_{k i}=\left(t_{k i 1}, \ldots, t_{k i M_{k i}}\right)^{T}$, $t_{k i j}=\omega_{k i j}, \boldsymbol{y}_{k i}=\left(y_{k i 1}, \ldots, y_{k i M_{k i}}\right)^{T}, \boldsymbol{y}_{k}=\left(\boldsymbol{y}_{k 1}, \ldots, \boldsymbol{y}_{k N_{k}}\right)^{T}$ and $\boldsymbol{Y}=\left(\boldsymbol{y}_{1}, \ldots, \boldsymbol{y}_{K}\right)$ for $k=$ $1,2, \ldots K, \quad i=1,2, \ldots, N_{k}$. The properties ii and iii of the Wiener processes implies that the Brownian movement $\boldsymbol{y}_{k i}$ follows a multivariate normal distribution with mean,

and covariance

$$
\mu_{k i}=\mu_{\eta} h_{k} \boldsymbol{T}_{k i}
$$

$$
\begin{aligned}
\boldsymbol{\Omega}_{k i} & =\sigma_{\eta}^{2} \widetilde{\boldsymbol{\Omega}}_{k i,} \\
\widetilde{\mathbf{H}}_{k i} & =\tilde{\kappa} h_{k}\left[\begin{array}{clll}
\omega_{k i 1} & \omega_{k i 1} & \ldots & \omega_{k i 1} \\
\omega_{k i 1} & \omega_{k i} & \ldots & \omega_{k i 2} \\
\vdots & \vdots & \ddots & \vdots \\
\omega_{k i 1} & \omega_{k i 2} & \ldots & \omega_{k i M_{k i}}
\end{array}\right]+\tilde{\sigma}_{\varepsilon}^{2} \boldsymbol{T}_{k i} \boldsymbol{T}_{k i}^{\mathbf{T}},
\end{aligned}
$$

where $\boldsymbol{I}_{k i}$ is the identity matrix of order $M_{k i}$, where $\tilde{k}=\frac{k}{\sigma_{\eta}^{2}}$ and $\tilde{\sigma}_{\epsilon}^{2}=\frac{\sigma_{\epsilon}^{2}}{\sigma_{\eta}^{2}}$ are a reparameterization to facilitate the inference. The log-likelihood function of unknown parameters $\widetilde{\Theta}=\left\{\mu_{\eta}, \sigma_{\eta}^{2}, \tilde{\kappa}, \beta, \tilde{\sigma}_{\varepsilon}^{2}\right\}$ is expresses as

$$
\begin{aligned}
l(\widetilde{\boldsymbol{\Theta}} \mid \boldsymbol{Y})=- & \frac{1}{2} \ln (2 \pi) \sum_{k=1}^{K} \sum_{i=1}^{N_{k}} M_{k i} \\
& -\frac{1}{2} \ln \left(\sigma_{\eta}^{2}\right) \sum_{k=1}^{K} \sum_{i=1}^{N_{k}} M_{k i}-\frac{1}{2} \sum_{k=1}^{K} \sum_{i=1}^{N_{k}} \ln \left|\widetilde{\boldsymbol{\Omega}}_{k i}\right| \\
& -\frac{1}{2 \sigma_{\eta}^{2}} \sum_{k=1}^{K} \sum_{i=1}^{N_{k}}\left(\boldsymbol{y}_{k i}-\mu_{\eta} h_{k} \boldsymbol{T}_{k i}\right)^{T} \widetilde{\boldsymbol{\Omega}}_{k i}^{-\mathbf{1}}\left(\boldsymbol{y}_{k i}-\mu_{\eta} h_{k} \boldsymbol{T}_{k i}\right) .
\end{aligned}
$$

In order to estimate the parameters $\mu_{\eta}$ and $\sigma_{\eta}^{2}$, the values of $\left(\tilde{\kappa}, \beta, \tilde{\sigma}_{\varepsilon}^{2}\right)$ are fixed to specific values, then the derivatives $\frac{\partial l(\widetilde{\boldsymbol{\Theta}} \mid \boldsymbol{Y})}{\partial \mu_{\eta}}$ and $\frac{\partial l(\widetilde{\boldsymbol{\Theta}} \mid \boldsymbol{Y})}{\partial \sigma_{\eta}^{2}}$ of (9) with respect to the parameters $\mu_{\eta}$ and $\sigma_{\eta}^{2}$ are computed and equated to zero, obtaining the following close -form expression to the maximum likelihood estimator MLE $\hat{\mu}_{\eta}$ and $\hat{\sigma}_{\eta}^{2}$ of $\mu_{\eta}$ and $\sigma_{\eta}^{2}$ 


$$
\begin{gathered}
\hat{\mu}_{\eta}=\frac{\sum_{k=1}^{K} \Sigma_{i=1}^{N_{k}} h_{k} \boldsymbol{T}_{k i}^{T} \widetilde{\mathbf{\Omega}}_{k i}^{-1} \boldsymbol{y}_{k i}}{\sum_{k=1}^{K} \Sigma_{i=1}^{N_{k}} h_{k}^{2} \boldsymbol{T}_{k i}^{T} \widetilde{\mathbf{\Omega}}_{k i}^{-1} \boldsymbol{T}_{k i}} \\
\hat{\sigma}_{\eta}^{2}=\frac{1}{\sum_{k=1}^{K} \Sigma_{i=1}^{N_{k}} M_{k i}} \sum_{k=1}^{K} \sum_{i=1}^{N_{k}}\left(\boldsymbol{y}_{k i}-\hat{\mu}_{\eta} h_{k} \boldsymbol{T}_{k i}\right)^{T} \widetilde{\boldsymbol{\Omega}}_{k i}^{-1}\left(\boldsymbol{y}_{k i}-\hat{\mu}_{\eta} h_{k} \boldsymbol{T}_{k i}\right) .
\end{gathered}
$$

Substituting (10) and (11) in (9) and simplifying in the log-likelihood function, it is obtained

$$
\begin{gathered}
l(\widetilde{\boldsymbol{\Theta}} \mid \boldsymbol{Y})=-\frac{1}{2}(\ln (2 \pi)+1) \sum_{k=1}^{K} \sum_{i=1}^{N_{k}} M_{k i} \\
-\frac{1}{2} \ln \left(\hat{\sigma}_{\eta}^{2}\right) \sum_{k=1}^{K} \sum_{i=1}^{N_{k}} M_{k i}-\frac{1}{2} \sum_{k=1}^{K} \sum_{i=1}^{N_{k}} \ln \left|\widetilde{\mathbf{H}}_{k i}+h_{k}^{2} \boldsymbol{T}_{k i} \boldsymbol{T}_{k i}^{\mathrm{T}}\right| .
\end{gathered}
$$

Note that the matrix $\widetilde{\mathbf{H}}_{k i}$ depends on the parameters $\tilde{\kappa}, \beta$ and $\tilde{\sigma}_{\varepsilon}^{2}$ and $h_{k}^{2}$ depends on $\beta$. Therefore, the maximum likelihood estimates of $\tilde{\kappa}, \beta, \tilde{\sigma}_{\varepsilon}^{2}$ can be obtained by maximizing the log-likelihood function (12) employing the L-BFGS-B quasi Newton optimization method that can be found in the R-project packages [37]. The value of $\hat{\kappa}$ can be obtained by the following equations:

$$
\begin{gathered}
\hat{\kappa}=\hat{\tilde{\kappa}} \cdot \hat{\sigma}_{\eta}^{2}, \\
\hat{\sigma}_{\varepsilon}^{2}=\hat{\tilde{\sigma}}_{\varepsilon}^{2} \cdot \hat{\sigma}_{\eta}^{2},
\end{gathered}
$$

One objective of the reliability analysis is to estimate the useful life of the product through the life distribution. To obtain the life distribution of a product using degradation data Li. et al [31] incorporate the measurement errors into the deduction of the expression for the CDF and PDF of the failure time

$$
T=\inf \{t: Y(t) \geq w\}
$$

where $T$ corresponds to the first time that the degradation process $Y$ hits a failure threshold $w$. They deduced the life PDF expression for each stress level $S_{k}$, which can be found in equation (12) at [31] Note that in this formulation they used two different time scales $\Lambda(t)$ and $\tau(t)$, when $\tau(t)=\Lambda(t)$, this PDF is reduced to,

$$
f_{S_{k}}(t)=\frac{\sigma_{\varepsilon}^{2} h_{k} \mu_{\eta}+\left(\kappa h_{k}+h_{k}^{2} \sigma_{\eta}^{2}\right) w}{\sqrt{2 \pi\left(h_{k} \omega\left(\kappa+h_{k} \sigma_{\eta}^{2} \omega\right)+\sigma_{\varepsilon}^{2}\right)^{3}}} \cdot \frac{d \omega}{d t} \cdot \exp \left\{\frac{-\left(w-h_{k} \mu_{\eta} \omega\right)^{2}}{2\left(h_{k} \omega\left(\kappa+h_{k} \sigma_{\eta}^{2} \omega\right)+\sigma_{\varepsilon}^{2}\right)}\right\}
$$

which is the one used in this work. Note $f_{S_{k}}(t)$ dependens on the parameters set $\widetilde{\boldsymbol{\Theta}}$.

\subsection{Methodology and Analysis of Degradation Data under the Wiener Process/ADT}

To obtain degradation data of GNPs@LA under a CSADT, several samples were synthesized following the methodology reported by Cornejo et al [19] and using $\mathrm{NaCl}$ as an acceleration factor. The stress test levels were based on an exploratory study, leaving three different levels of effort for three different populations. UV-Vis absorption spectra of colloids were carried out every third day generating degradation signals for low, medium, and high levels. To this study the material degradation was determined considering the absorbance between 450 and $550 \mathrm{~nm}$, and the maximum characteristic peak of gold. Gold colloids with a characteristic peak greater than 525 were considered as failure.

Since this work proposes to estimate the life distribution of GNPs@LA applying a CSADT. Accordingly, the following can be defined: 
- $\quad$ The percentage levels of $\mathrm{NaCl}$ are indexed as $k=1, \ldots, K$

- $\quad$ The population of samples are indexed as $i=1, \ldots, N_{k}$

- $\quad$ Measurement times are indexed as $j=1, \ldots, M_{k i}$

Once the degradation signals have been defined as degradation data, we proceed to obtain the configuration of the CSADT that describes the degradation trajectories. Thus, this work proposes the non-linear Wiener process with drift parameter, random effects, measurement errors and different link functions in the covariability (Table 1, Section 2.3) is used. More specifically the model assumes:

- A drift parameter with transformed times $\omega_{k i j}=\Lambda\left(t_{k i j}\right)=\left(t_{k i j}\right)^{b}$ that explain the non-linearity of the data.

- Random effects in the drift parameter. Due to different units have different drift parameters while all diffusion parameters have the same value under a certain effort. So, the parameter $\eta$ in the drift term at (5) is assumed to has a normal distribution $\eta \sim N\left(\mu_{\eta}, \sigma_{\eta}^{2}\right)$.

- $\quad$ Measurement errors with $\varepsilon_{k i j} \sim N\left(0, \sigma_{\varepsilon}^{2}\right)$.

- Covariability, which is expressed via the link function $h_{k}$, where the accelerated model is immersed. It is highlighted three accelerated models that were evaluated in this work corresponding to, inverse power, linear model, and the quadratic model, see (Table 1, Section 2.3).

Now, proposing the CSADT and the Wiener process with the aforementioned characteristics, the degradation process can be stablished as formula (5). Thus, the following parameter set $\widetilde{\boldsymbol{\Theta}}=\left\{\mu_{\eta}, \sigma_{\eta}^{2}, \tilde{\kappa}, \beta, \tilde{\sigma}_{\varepsilon}^{2}\right\}$ will be estimated to get the life distribution. We remark that $\beta=\left(\beta_{1}, \beta_{2}\right)$ in the quadratic model case and $\beta=\beta_{1}$ in the remaining cases.

All the above were programmed in the statistical software R, where the MLE was applied to obtain the parameters set $\widetilde{\boldsymbol{\Theta}}$. Figure 2 shows the proposed methodology.

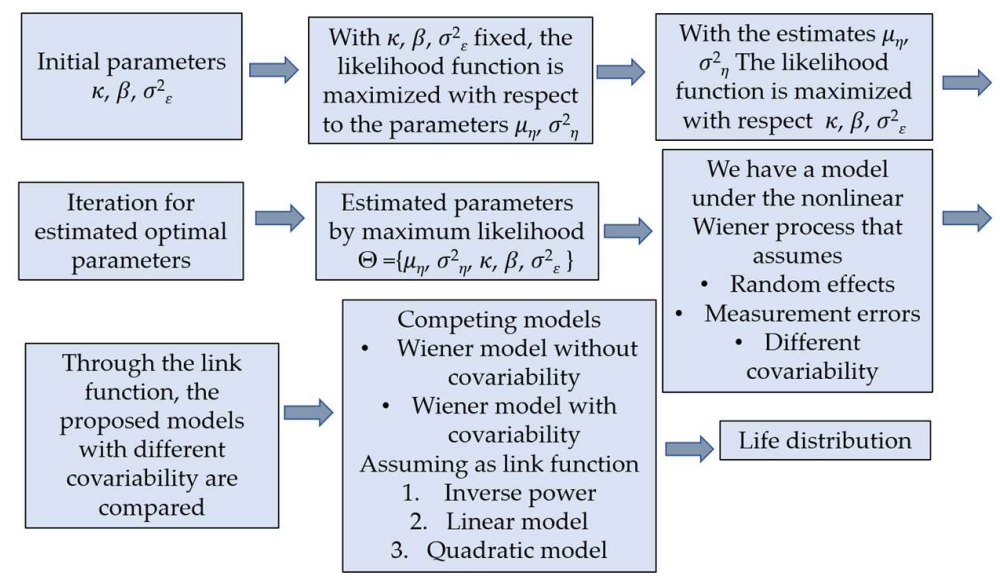

Figure 2. Methodology for the estimation of parameters in the proposed models.

As can be seen in Figure 2, different degradation models have been proposed. To select the best model will be used the AIC criterion [38] and for the evaluation and validation, the estimated parameters of the model will be employed and the Bootstraping distribution [39] will be calculated with the construction of confidence intervals.

\section{Results}


The constant stress ADT in GNPs@LA had 3 levels, these being: 19 samples for the low level with $8 \% \mathrm{NaCl}, 13$ samples for the medium level with $12 \%$ and 12 samples for the high level with $16 \% \mathrm{NaCl} \mathrm{m} / \mathrm{v}$. With a censorship time of 69 days; except to the low level which was 51 due to remaining time conditions and modifications of the plan. The degradation measurements were performed every third day generating a total of 18 measurements for low level and 23 measurements for medium level and high level, providing degradation signals over time.

From UV-Vis absorption spectra degradation signals were obtained. In Figure 3, the changes of the spectra are graphically presented comparing the first measurement and the last measurement in the range of 400 to $800 \mathrm{~nm}$ in wavelength.

(a)
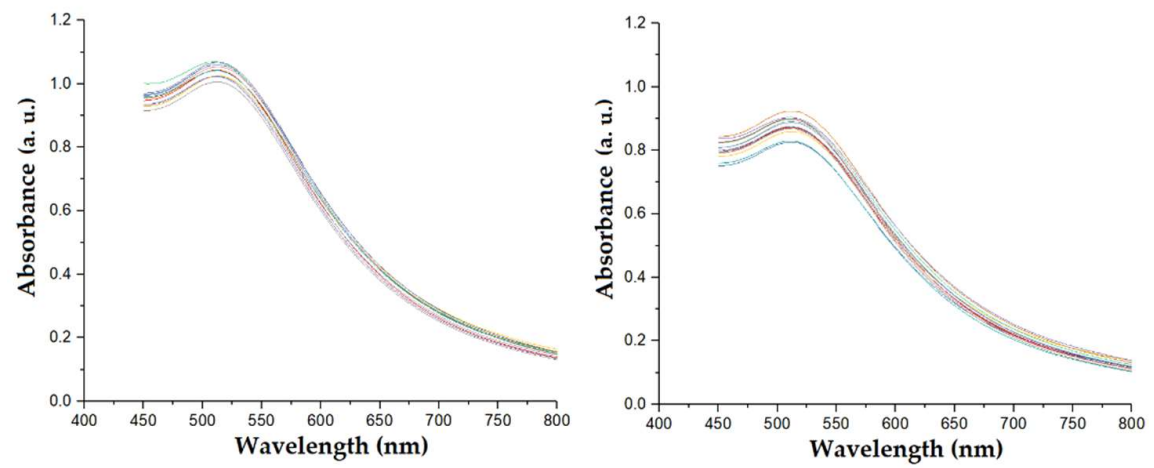

(b)
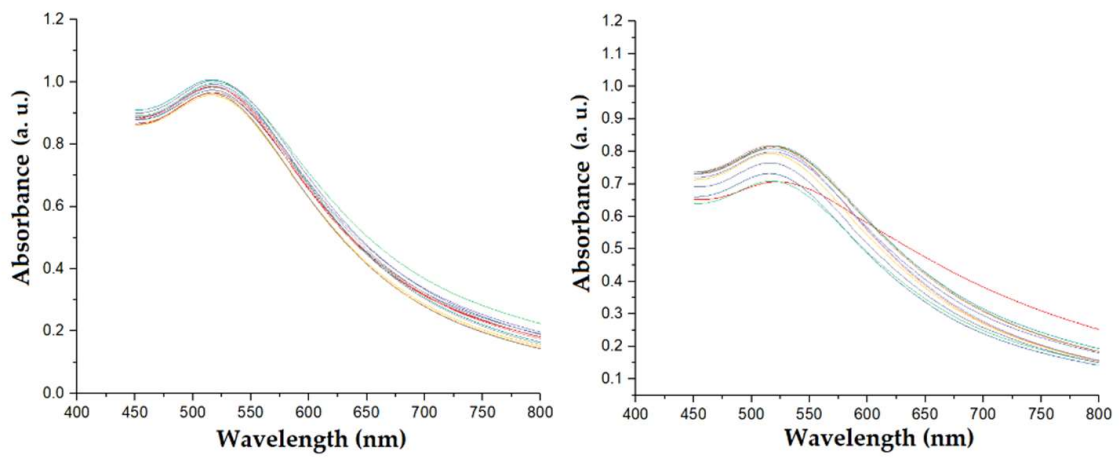

(c)
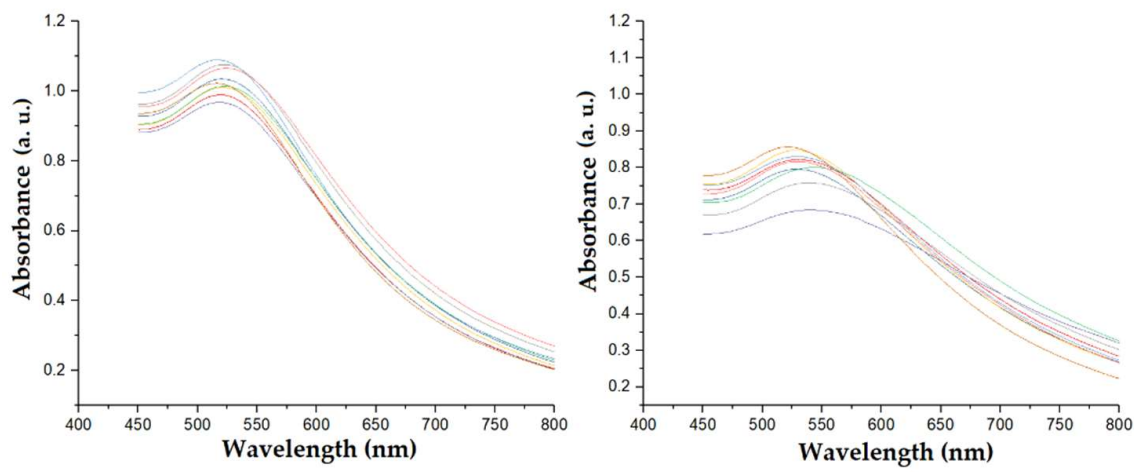

Figure 3. UV-Vis absorption spectra of GNPS@LA. Initial (left) and final characterization (right) for the ADT: (a) low level, $8 \% \mathrm{NaCl}$; (b) medium level, $12 \% \mathrm{NaCl}$; and (c) high level, $16 \% \mathrm{NaCl}$. 
It can be observed in Figure 3 that for the three degradation levels, the absorbance amplitude decreases, and the width of the band broadens causing a red shifted of characteristic peak due to their increase in size and the aggregation of the gold nanoparticles. It can be also noticed that at higher percentages of $\mathrm{NaCl}$ the degradation is more appreciable than at lower percentages. To have a better relationship between UV-Vis spectra and degradation material we made a graph from the average of initial and final characterization for each level, which can be seen in Figure 4. The area comprised between 450 and $550 \mathrm{~nm}$ from UV-Vis spectra was used to quantify the material degradation. Additionally, when the characteristic peak moves above $525 \mathrm{~nm}$ is considered as the failure threshold.
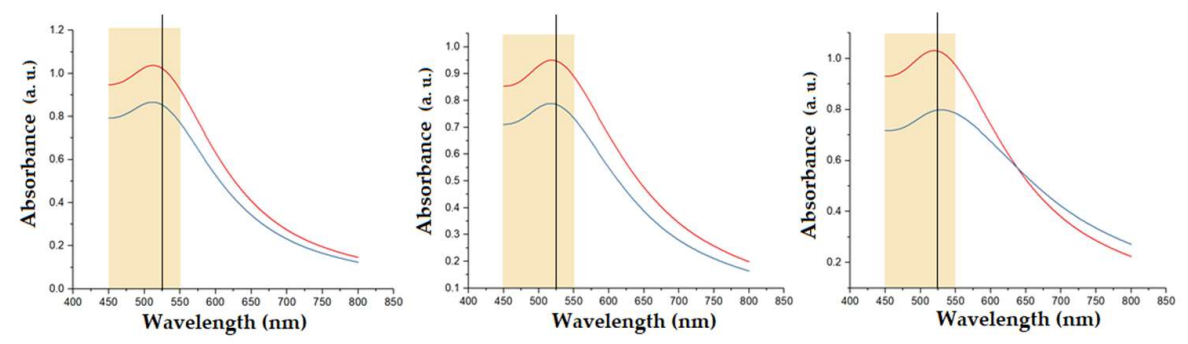

Initial measurement $\_$Final measurement

Figure 4. Average UV-Vis absorption spectra from the initial and final characterization for each level of the ADT. From left to right: low, medium, and high level, respectively.

From Figure 4, a change in area between the initial and final characterization is well noted and it was used to obtain degradation data.

To maintain a notation according to the property of independent increments and a normal distribution under the Wiener process, the area for each measurement was calculated, and each degradation increment was obtained from the difference between the first day area and the subsequent day areas, these were the increments of degradation to be modeled. Under this consideration, the degradation trajectories were obtained for each sample at the different levels of $\mathrm{NaCl}$.

In Figure 5 the different trajectories at each level are shown, it can also be observed that there is a non-monotonous behavior with increasing and decreasing trends. Also, it is observed that the degradation of gold colloids with same levels of $\mathrm{NaCl}$ are different, this can be attributed to unobservable factors such as concentration, unit-to-unit variability, inherent randomness, as well as the measurement variability of each sample. Thus, the Wiener stochastic model was chosen with its four variants since it has great potential to capture stochastic dynamics, and it is also applicable to non-monotonic impairments, providing a satisfactory and flexible description of the impairment data.
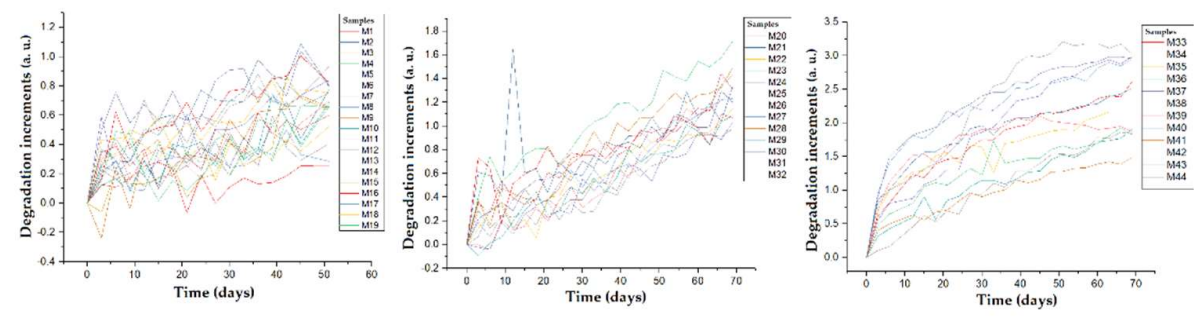

Figure 5. Degradation trajectories of gold colloids for the different levels of $\mathrm{NaCl}: 8 \%, 12 \%$, and $16 \%$ of $\mathrm{ADT}$, from left to right.

For this study, we propose to model the degradation trajectories under the non-linear Wiener process, with random effects, error measurements and different covariability using three different link functions, which generates three different models. 
To obtain the optimal parameters in each model, the initial parameters should be close to the true model parameter to be estimated, as well as the value $b$ in the time transformation, these were obtained by a preliminary package made by us using RStudio that performs an individual regression using least squares for each degradation path giving as initial parameters $b=0.61, \tilde{\kappa}=0.65, \beta=-0.8479, \tilde{\sigma}_{\varepsilon}^{2}=.01$ assuming inverse power, for a linear model they were $b=0.61, \tilde{\kappa}=0.65, \beta=-0.18, \tilde{\sigma}_{\varepsilon}^{2}=.01$ and finally for a quadratic model the initial parameter were $b=0.61, \tilde{\kappa}=0.65, \beta_{1}=0.04, \beta_{2}=0.1 \tilde{\sigma}_{\varepsilon}^{2}=$ .01 . Once these initial parameters were estimated, they were fixed at the likelihood function to estimate the optimal values of $\mu_{\eta}$ and $\sigma_{\varepsilon}^{2}$ via the MLE approach (in closed form (10) and (11) ) presented in Section 2.4. Scale time transformation parameter was remained fixed in $b=0.61$ during optimization and to avoid over-estimation parameter $\beta_{2}$, the constraint $\mu_{\eta} h_{k}>\mu_{\eta} h_{k+1}$ for $k>1$ was added in the quadratic model. The obtained optimal parameters are highlighted in Table 2.

Table 2. Obtained Optimal parameters for each accelerated degradation model.

\begin{tabular}{cccccc}
\hline \multirow{2}{*}{ Covariability } & \multicolumn{5}{c}{ Optimal parameters } \\
\cline { 2 - 6 } & $\mu_{\eta}$ & $\sigma_{\eta}^{2}$ & $\kappa$ & $\beta$ & $\tilde{\sigma}_{\varepsilon}^{2}$ \\
\hline Inverse Power & 0.00251 & $4.0 \mathrm{E}-08$ & 0.00028 & -1.4986 & 0.00801 \\
& & & & & \\
Linear Model & 0.01741 & $2.0 \mathrm{E}-07$ & 0.00181 & -0.1428 & 0.00849 \\
Quadratic Model & 0.04386 & $6.4 \mathrm{E}-06$ & 0.00460 & $(0.014,0.0062)$ & 0.00838 \\
& & & & & \\
\hline
\end{tabular}

With the estimated parameters of the model in Table 2 and establishing $w$ as the failure threshold, which corresponds to a 1.51 degradation increment, equivalent to an area of 2.1 units implying a $525 \mathrm{~nm}$ deface in the wavelength. The life distribution is given by (16). Therefore, the density and cumulative density functions for each degradation level are shown in Figure 6. It is observed that at higher percentages the degradation is more noticeable, also the probability mass concentrates more towards zero as the percentage of $\mathrm{NaCl}$ increases, in accordance with the accelerated degradation test.

(a)

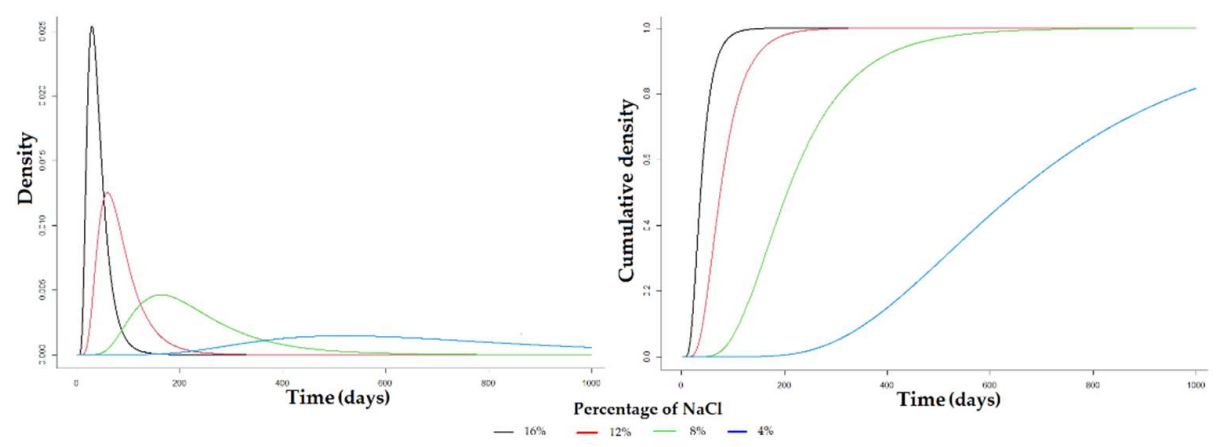

(b) 


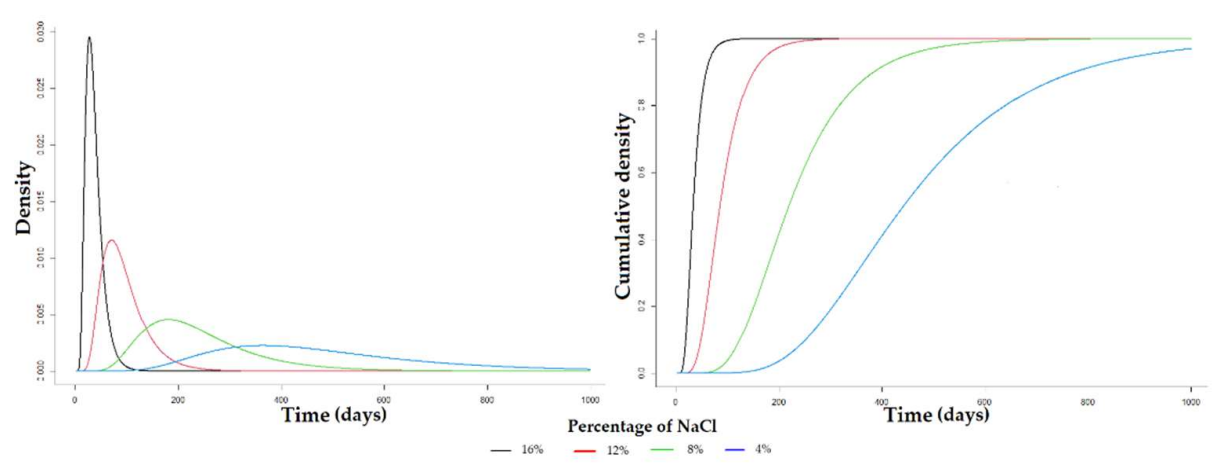

(c)

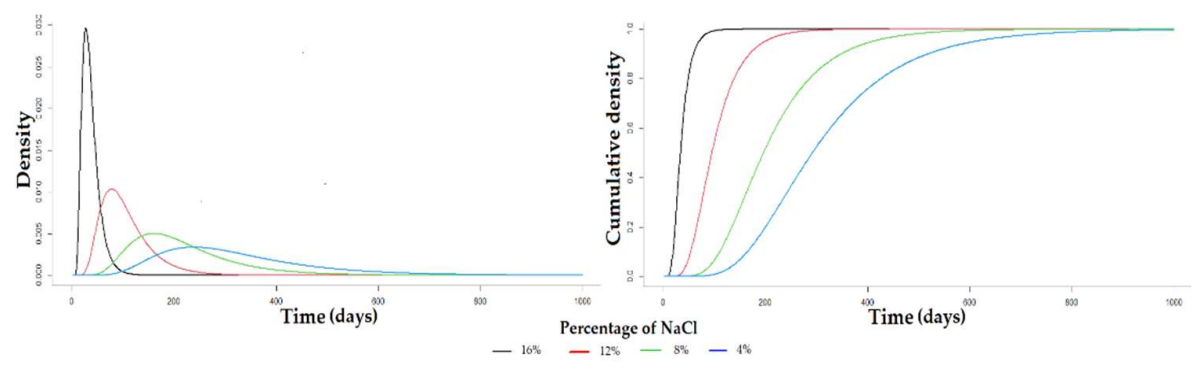

Figure 6. Density and cumulative density for each proposed model for different percentages of $\mathrm{NaCl}$ assuming a covariability of: (a) inverse power; (b) linear model; (c) quadratic model.

Given the cumulative density of the model, this can be evaluated to make desired inferences and consequently estimate the useful life under different conditions. As an example of this, Table 3 presents some failure rates based on formula (16).

Table 3. Failure rate in gold colloids with different $\mathrm{NaCl}$ percentages and covariability obtained by the three proposed models.

\begin{tabular}{|c|c|c|c|c|c|c|c|c|c|}
\hline \multirow{2}{*}{ Days } & \multicolumn{9}{|c|}{ Inverse power model } \\
\hline & $0 \%$ & $1 \%$ & $5 \%$ & $8 \%$ & $10 \%$ & $12 \%$ & $15 \%$ & $16 \%$ & $20 \%$ \\
\hline 30 & Undefined & 0.000 & 0.000 & 0.000 & 0.001 & 0.023 & 0.211 & 0.321 & 0.756 \\
\hline 72 & Undefined & 0.000 & 0.000 & 0.012 & 0.144 & 0.457 & 0.853 & 0.916 & 0.994 \\
\hline 360 & Undefined & 0.000 & 0.103 & 0.882 & 0.990 & 0.999 & 1.000 & 1.000 & 1.000 \\
\hline 720 & Undefined & 0.002 & 0.585 & 0.996 & 1.000 & 1.000 & 1.000 & 1.000 & 1.000 \\
\hline \multirow[t]{3}{*}{1080} & Undefined & 0.013 & 0.857 & 1.000 & 1.000 & 1.000 & 1.000 & 1.000 & 1.000 \\
\hline & \multicolumn{9}{|c|}{ Linear model } \\
\hline & $0 \%$ & $1 \%$ & $5 \%$ & $8 \%$ & $10 \%$ & $12 \%$ & $15 \%$ & $16 \%$ & $20 \%$ \\
\hline 30 & 0.000 & 0.000 & 0.000 & 0.000 & 0.000 & 0.009 & 0.216 & 0.399 & 0.971 \\
\hline 72 & 0.000 & 0.000 & 0.000 & 0.006 & 0.073 & 0.346 & 0.886 & 0.960 & 1.000 \\
\hline 360 & 0.001 & 0.020 & 0.322 & 0.872 & 0.987 & 1.000 & 1.000 & 1.000 & 1.000 \\
\hline 720 & 0.062 & 0.315 & 0.868 & 0.997 & 1.000 & 1.000 & 1.000 & 1.000 & 1.000 \\
\hline \multirow[t]{3}{*}{1080} & 0.268 & 0.669 & 0.980 & 1.000 & 1.000 & 1.000 & 1.000 & 1.000 & 1.000 \\
\hline & \multicolumn{9}{|c|}{ Quadratic model } \\
\hline & $0 \%$ & $1 \%$ & $5 \%$ & $8 \%$ & $10 \%$ & $12 \%$ & $15 \%$ & $16 \%$ & $20 \%$ \\
\hline 30 & 0.000 & 0.000 & 0.000 & 0.000 & 0.000 & 0.005 & 0.191 & 0.413 & 0.998 \\
\hline
\end{tabular}




\begin{tabular}{cccccccccc}
\hline 72 & 0.000 & 0.000 & 0.001 & 0.013 & 0.065 & 0.270 & 0.859 & 0.958 & 1.000 \\
360 & 0.622 & 0.611 & 0.733 & 0.913 & 0.981 & 0.999 & 1.000 & 1.000 & 1.000 \\
720 & 0.969 & 0.966 & 0.985 & 0.998 & 1.000 & 1.000 & 1.000 & 1.000 & 1.000 \\
1080 & 0.997 & 0.997 & 0.999 & 1.000 & 1.000 & 1.000 & 1.000 & 1.000 & 1.000 \\
\hline
\end{tabular}

As can be seen on Table 3, results between different models differ, being the quadratic model, which provides the lowest failure rate. To select the best model, and establishing that covariability influences the modeling, the Akaike's information criterion (AIC) was applied. Modeling without covariability an AIC value of -931.703 is obtained which is greater compared with the AIC values for the proposed models, as can be seen on table 4.

Table 4. AIC criterion values for the different models considering covariability

\begin{tabular}{ccc}
\hline Inverse power model & Linear model & Quadratic model \\
\hline-993.771 & -997.439 & -998.017 \\
\hline
\end{tabular}

From Table 4, it can readily see that covariability has an influence on the models and must be embedded in the process. The application of the AIC criterion suggests that quadratic model is a better option for the degradation data obtained. Continuing with the assessment, the estimated parameters are used and with this confidence intervals (CI) are calculated through the Bootstrap method using 4000 datasets [40]. The results are showed in Figure 7 for each model.

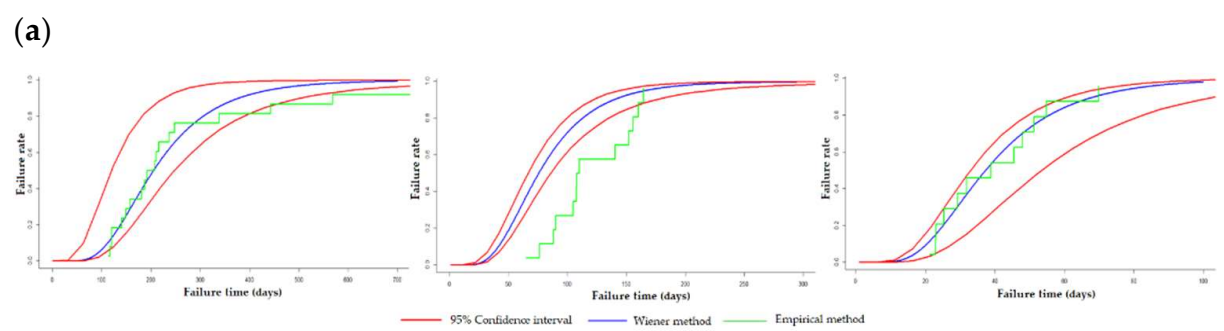

(b)

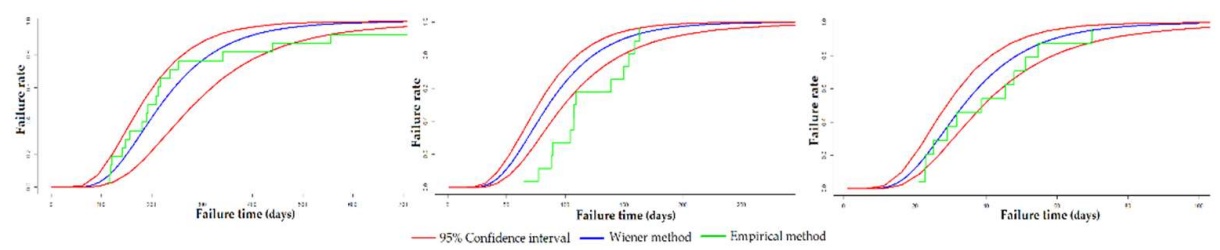

(c)

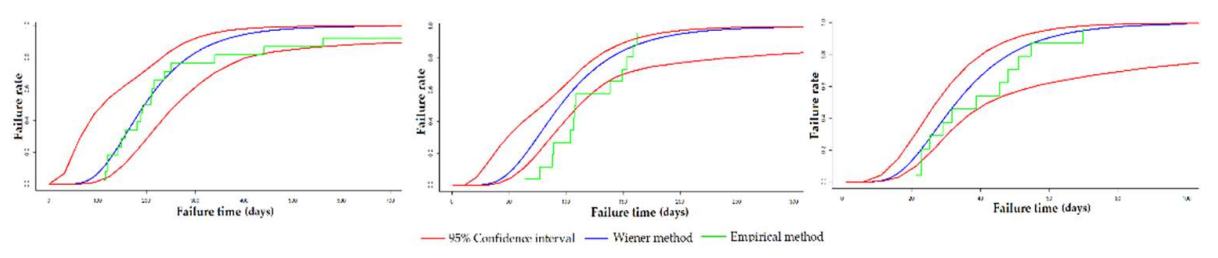

Figure 7. Bootstrap results for each degradation model using $\mathrm{NaCl}$ at $8 \%, 12 \%$ and $16 \%$, from left to right. (a) Inverse power model; (b) Linear model; (c) Quadratic model.

According to the CI with a confidence level of 95\% which are observed in Figure 7, the percentages of $8 \%$ and $16 \%$ present an empirical value which is closed to the 
theoretical cumulative density. On the other hand, it can be noted that at $12 \%$ percentage the three models present several distant points outside the CI, however, the quadratic model seems to have more values within the confidence interval, as well as close to the theoretical distribution.

\section{Discussion}

The results of the research show that according to the AIC criterion and the bootstrap confidence interval the quadratic acceleration model has a better adjustment of the degradation of the GNPs@LA, however, in the ADT and ALT the samples usually show a degree of curvature that is not sufficient for the AIC criterion to better consider the quadratic acceleration model over a linear one. In our model, the curvature was large enough to improve the AIC criterion. When a curvature parameter is added in an ADT, special attention should be paid, since there could be an overestimation of the degradation of the product under normal conditions of use, in order to avoid this overestimation we add the restriction $\mu_{\eta} h_{k}>\mu_{\eta} h_{k+1}$ for $k>1$ in the estimation of the model parameters, thus obtaining an estimate of the failure fraction of the GNPs@LA under normal conditions of use consistent with the little knowledge that we had of them. We also recommend further investigation of the quadratic acceleration model in ADT tests.

\section{Conclusions}

This research proposed a methodology and an analysis model to estimate the failure rate and useful life of GNPs@LA based on accelerated degradation tests and non-linear Wiener process incorporating random effects, error measures and covariability. The proposed scheme employs three different link functions in covariability using the inverse power, linear and quadratic models.

The modeling has been tested using $\mathrm{NaCl}$ as an acceleration factor and a three-level constant stress ADT with $8 \%, 12 \%$ and $16 \%$ of $\mathrm{NaCl}$ as degradation signals and as degradation data in the Wiener process. The data presented a non-monotonous behavior with oscillatory tendencies, and the GNPs@LA degradation observed for the same population was different thus the Wiener stochastic process was applied with its four variants.

It is demonstrated that the model applied by the non-linear Wiener process, with random effects, error measures and covariability that uses the quadratic model as a link function was the most effective and gives the best estimate of the degradation rate of the shelf life of GNP@LA and as a function of $\mathrm{NaCl}$. These results can be used to provide guarantees of commercially available nanomaterials.

Supplementary Materials: Not applicable.

Author Contributions: Conceptualization, B.S.-S., B.M., D.C.-M., and R.M.-A.; methodology, B.S.S., B.M., D.C.-M., and R.M.-A.; software, B.S.-S., B.M., D.C.-M., and R.M.-A.; writing-original draft preparation, B.S.-S., B.M., D.C.-M., and R.M.-A.; validation, B.S.-S., B.M., D.C.-M., and R.M-A; formal analysis, B.S.-S., B.M., D.C.-M., R.M.-A. and V.C.-M.; investigation, B.S.-S., B.M., D.C.-M., R.M.A. and V.C.-M.; data curation, B.S.-S., B.M., D.C.-M., and R.M.-A.; writing - review and editing, B.S.S., B.M., D.C.-M., R.M.-A. and V.C.-M.; supervision, B.S.-S., B.M., D.C.-M., R.M.-A. and V.C.-M.; project administration, B.S.-S., B.M., D.C.-M., R.M.-A. and V.C.-M.; funding acquisition, B.S.-S., B.M., D.C.-M., R.M.-A. and V.C.-M. All authors have read and agreed to the published version of the manuscript.

Funding: This research received no external funding.

Institutional Review Board Statement: Not applicable.

Informed Consent Statement: Not applicable. 
Data Availability Statement: The data presented in this work are available on request from the corresponding author.

Acknowledgments: The authors wish to express the acknowledgement to CONACYT that supported this research.

Conflicts of Interest: The authors declare no conflict of interest, whatsoever.

\section{References}

1. Ye, Z.-S.; Xie, M. Stochastic modelling and analysis of degradation for highly reliable products. Appl. Stoch. Models Bus. Ind. 2015, 31, 16-32, doi:10.1002/asmb.2063.

2. Zhang, Z.; Si, X.; Hu, C.; Lei, Y. Degradation data analysis and remaining useful life estimation: a review on Wiener-process-based methods. Eur. J. Oper. Res. 2018, 271, 775-796, doi:10.1016/j.ejor.2018.02.033.

3. Cornejo-Monroy, D.; Acosta-Torres, L.S.; Moreno-Vega, A.I.; Saldana, C.; Morales-Tlalpan, V.; Castaño, V.M. Gold nanostructures in medicine: past, present and future. J. Nanosci. Lett. 2013, 3, 1-9.

4. Larsson, S.; Jansson, M.; Boholm, Å. Expert stakeholders' perception of nanotechnology: risk, benefit, knowledge, and regulation. J. Nanopart. Res. 2019, 21, doi:10.1007/s11051-019-4498-1.

5. Saldivar-Tanaka, L. Regulando la nanotecnología. Mundo nano (en línea) 2018, 12, 37-57, doi:10.22201/ceiich.24485691e.2019.22.63140.

6. Haume, K.; Rosa, S.; Grellet, S.; Śmiałek, M.A.; Butterworth, K.T.; Solov'yov, A.V.; Prise, K.M.; Golding, J.; Mason, N.J. Gold nanoparticles for cancer radiotherapy: a review. Cancer Nanotechnol. 2016, 7, 8, doi:10.1186/s12645-016-0021-x.

7. Tamayo, L.; Palza, H.; Bejarano, J.; Zapata, P.A. Polymer composites with metal nanoparticles: synthesis, properties, and applications. In Polymer Composites with Functionalized Nanoparticles, Pielichowski, K., Majka, T.M., Eds.; Elsevier: 2019; pp. 249-286.

8. Huang, K.; Ma, H.; Liu, J.; Huo, S.; Kumar, A.; Wei, T.; Zhang, X.; Jin, S.; Gan, Y.; Wang, P.C.; et al. Sizedependent localization and penetration of ultrasmall gold nanoparticles in cancer cells, multicellular spheroids, and tumors in vivo. ACS Nano 2012, 6, 4483-4493, doi:10.1021/nn301282m.

9. Afonso, A.S.; Pérez-López, B.; Faria, R.C.; Mattoso, L.H.C.; Hernández-Herrero, M.; Roig-Sagués, A.X.; Maltez-da Costa, M.; Merkoçi, A. Electrochemical detection of Salmonella using gold nanoparticles. Biosens. Bioelectron. 2013, 40, 121-126, doi:10.1016/j.bios.2012.06.054.

10. Guo, J.; Rahme, K.; He, Y.; Li, L.L.; Holmes, J.D.; O'Driscoll, C.M. Gold nanoparticles enlighten the future of cancer theranostics. Int. J. Nanomedicine 2017, 12, 6131-6152, doi:10.2147/ijn.S140772.

11. Sivasankarapillai, V.S.; Pillai, A.M.; Rahdar, A.; Sobha, A.P.; Das, S.S.; Mitropoulos, A.C.; Mokarrar, M.H.; Kyzas, G.Z. On facing the SARS-CoV-2 (COVID-19) with combination of nanomaterials and medicine: possible strategies and first challenges. Nanomaterials 2020, 10, 852.

12. Daraee, H.; Eatemadi, A.; Abbasi, E.; Fekri Aval, S.; Kouhi, M.; Akbarzadeh, A. Application of gold nanoparticles in biomedical and drug delivery. Artif. Cells, Nanomed., Biotechnol. 2016, 44, 410-422, doi:10.3109/21691401.2014.955107.

13. Parveen, S.; Misra, R.; Sahoo, S.K. Nanoparticles: a boon to drug delivery, therapeutics, diagnostics and imaging. Nanomedicine 2012, 8, 147-166, doi:10.1016/j.nano.2011.05.016.

14. Patra, S.; Mukherjee, S.; Barui, A.K.; Ganguly, A.; Sreedhar, B.; Patra, C.R. Green synthesis, characterization of gold and silver nanoparticles and their potential application for cancer therapeutics. Mater. Sci. Eng. C Mater. Biol. Appl. 2015, 53, 298-309, doi:10.1016/j.msec.2015.04.048. 
15. Shilo, M.; Sharon, A.; Baranes, K.; Motiei, M.; Lellouche, J.-P.M.; Popovtzer, R. The effect of nanoparticle size on the probability to cross the blood-brain barrier: an in-vitro endothelial cell model. J. Nanobiotechnol. 2015, 13, 19, doi:10.1186/s12951-015-0075-7.

16. Li, J.; Li, J.E.J.; Zhang, J.; Wang, X.; Kawazoe, N.; Chen, G. Gold nanoparticle size and shape influence on osteogenesis of mesenchymal stem cells. Nanoscale 2016, 8, 7992-8007, doi:10.1039/C5NR08808A.

17. Corzo Lucioni, A. Síntesis de nanopartículas de oro obtenidas por reducción de H[AuCl4]. Rev. Soc. Quím. Perú 2012, 78, 79-90.

18. Tseng, K.-H.; Hsieh, C.-L.; Huang, J.-C.; Tien, D.-C. The effect of NaCl/pH on colloidal nanogold produced by pulsed spark discharge. J. Nanomater. 2015, 2015, 612324, doi:10.1155/2015/612324.

19. Cornejo-Monroy, D.; Sánchez-Santamaria, B.; Martínez-Gómez, E.A.; Olivas-Armendáriz, I.; VillanuevaMontellano, A.; Arias-Cerón, J.S.; Castaño, V.M. Facile synthesis of ultrasmall, highly stable, and biocompatible gold nanoparticles stabilized with lipoic acid: cytotoxicity and structural characterization. Nanotechnol. Russia 2019, 14, 607-612, doi:10.1134/S199507801906003X.

20. Mondragón, R.; Juliá, J.E.; Barba, A.; Jarque, J.C. Preparación y caracterización de nanofluidos: influencia de variables sobre su estabilidad, estado de aglomeración y propiedades físicas. Bol. Soc. Esp. Ceram. Vidr. 2014, 53, 101-110, doi:10.3989/cyv.142014.

21. Ramdayal; Balasubramanian, K. Antibacterial application of polyvinylalcohol-nanogold composite membranes. Colloids Surf., A 2014, 455, 174-178, doi:10.1016/j.colsurfa.2014.04.050.

22. Yang, Y.; Matsubara, S.; Nogami, M.; Shi, J. Controlling the aggregation behavior of gold nanoparticles. Mater. Sci. Eng. B 2007, 140, 172-176, doi:10.1016/j.mseb.2007.03.021.

23. Haiss, W.; Thanh, N.T.K.; Aveyard, J.; Fernig, D.G. Determination of size and concentration of gold nanoparticles from UV-Vis spectra. Anal. Chem. 2007, 79, 4215-4221, doi:10.1021/ac0702084.

24. Martínez, J.C.; Chequer, N.A.; González, J.L.; Cordova, T. Alternative metodology for gold nanoparticles diameter characterization using PCA technique and UV-VIS spectrophotometry. Nanosci. Nanotechnol. 2012, 2, 184-189, doi:10.5923/j.nn.20120206.06.

25. Hermanson, G.T. Bioconjugate techniques, 3rd ed.; Academic Press: Rocford, 2013; p. 1200.

26. Meeker, W.Q.; Escobar, L.A. Statistical methods for reliability data, 1st ed.; Wiley: 1998; p. 712.

27. Liao, H.; Elsayed, E.A. Reliability inference for field conditions from accelerated degradation testing. Nav. Res. Logist. 2006, 53, 576-587, doi:10.1002/nav.20163.

28. Wang, X. Wiener processes with random effects for degradation data. J. Multivar. Anal. 2010, 101, 340-351, doi:10.1016/j.jmva.2008.12.007.

29. Whitmore, G.A.; Crowder, M.J.; Lawless, J.F. Failure inference from a marker process based on a bivariate Wiener model. Lifetime Data Anal. 1998, 4, 229-251, doi:10.1023/A:1009617814586.

30. Ye, Z.-S.; Shen, Y.; Xie, M. Degradation-based burn-in with preventive maintenance. Eur. J. Oper. Res. 2012, 221, 360-367, doi:10.1016/j.ejor.2012.03.028.

31. Li, J.; Wang, Z.; Zhang, Y.; Fu, H.; Liu, C.; Krishnaswamy, S. Degradation data analysis based on a generalized Wiener process subject to measurement error. Mech. Syst. Signal Process. 2017, 94, 57-72, doi:10.1016/j.ymssp.2017.02.031.

32. Peng, C.-Y.; Tseng, S.-T. Mis-specification analysis of linear degradation models. IEEE Trans. Reliab. 2009, 58, 444-455, doi:10.1109/TR.2009.2026784. 
33. Si, X.-S.; Wang, W.; Hu, C.-H.; Chen, M.-Y.; Zhou, D.-H. A Wiener-process-based degradation model with a recursive filter algorithm for remaining useful life estimation. Mech. Syst. Signal Process. 2013, 35, 219-237, doi:10.1016/j.ymssp.2012.08.016.

34. Si, X.-S.; Wang, W.; Hu, C.-H.; Zhou, D.-H.; Pecht, M.G. Remaining useful life estimation based on a nonlinear diffusion degradation process. IEEE Trans. Reliab. 2012, 61, 50-67, doi:10.1109/TR.2011.2182221.

35. Tsai, C.-C.; Tseng, S.-T.; Balakrishnan, N. Mis-specification analyses of gamma and Wiener degradation processes. J. Stat. Plan. Inference 2011, 141, 3725-3735, doi:10.1016/j.jspi.2011.06.008.

36. Sun, L.; Gu, X.; Song, P. Accelerated degradation process analysis based on the nonlinear Wiener process with covariates and random effects. Math. Probl. Eng. 2016, 2016, 5246108, doi:10.1155/2016/5246108.

37. Nocedal, J. Updating quasi-Newton matrices with limited storage. Math. Comput. 1980, 35, 773-782, doi:10.2307/2006193.

38. Nguyen, K.T.P.; Fouladirad, M.; Grall, A. Model selection for degradation modeling and prognosis with health monitoring data. Reliab. Eng. Syst. Saf. 2018, 169, 105-116, doi:10.1016/j.ress.2017.08.004.

39. Hesterberg, T.C. Bootstrap tilting confidence intervals and hypothesis test. 1999.

40. Jain, A.K.; Dubes, R.C.; Chen, C.-C. Bootstrap techniques for error estimation. IEEE Trans. Pattern. Anal. Mach. Intell. 1987, PAMI-9, 628-633, doi:10.1109/TPAMI.1987.4767957. 